\title{
On the understanding of current-induced spin polarization of three-dimensional topological insulators
}

\author{
Jifa Tian (1) 1,2,3, Seokmin Hong $2,4,5$, Shehrin Sayed ${ }^{2,4,6}$, Joon Sue Lee (i) ${ }^{7}$, Supriyo Datta ${ }^{2,4}$, Nitin Samarth (1) ${ }^{7}$ \& \\ Yong P. Chen ${ }^{1,2,4,8,9}$
}

ARISING FRom C. H. Li et al. Nature Communications https://doi.org/10.1038/ncomms13518 (2016).

$\mathrm{n}$ a recent $\operatorname{article}^{1}$, Li et al. reported the current-induced spin polarization (CISP) on topological insulator (TI) $\mathrm{Bi}_{2} \mathrm{Se}_{3}$ and on InAs (100) samples by spin potentiometry and compared the sign of the measured signals using a theoretical model to conclude the origin of the observed CISP in their TIs. Spin potentiometry has been used to electrically measure CISP in spin-orbital coupled (SOC) materials, such as TIs ${ }^{2-8}$, and semiconductor-based twodimensional electron gases $(2 \mathrm{DEG})^{9-11,12}$, where a ferromagnetic (FM) voltage probe with magnetization (M) collinear to the induced non-equilibrium spins is used to determine the CISP. The theoretical model by Li et al. consists of two key components: (a) a spin-dependent electrochemical potential diagram and (b) an argument that FM probe with magnetization along up (down) spin direction will measure the down (up) spin electrochemical potential. However, we bring attention to the inconsistencies in their paper. First, their (spatially varying) spin-dependent electrochemical potential diagram is incorrect and inconsistent with their topological surface states (TSS) band diagram to reflect the same experimental condition. The corrected potential diagram in conjunction with their model argument (b) is inconsistent with their assigned origin of CISP based on the sign of the measured signal. Second, they incorrectly stated that the experiment on $(\mathrm{Bi}$, $\mathrm{Sb})_{2} \mathrm{Te}_{3}$ reported by Lee et al. ${ }^{7}$ gave the same sign as their measurements, whereas it is opposite. Finally, we point out that the comparison of the sign of the measured signal from TI with that of InAs may not be sufficient to draw a conclusion on the origin of CISP in their measurements.

Li et al. ${ }^{1}$ adopted a model similar to our prior model ${ }^{5,13,14}$ to explain their observed CISP in terms of the spin-momentum locking (SML) of TSS (see Fig. 1). However, there is a qualitative difference regarding the electrochemical potentials between the model described by Li et al. in ref. ${ }^{1}$ (Fig. 1b, $\mathrm{c}$ taken from Fig. 5 of ref. ${ }^{1}$ ) and our model (Fig. 1d, e). First, their electrochemical potential diagram (see Fig. 1c) is inconsistent with their band diagram (see Fig. 1b) which is supposed to reflect the same experimental situation (see Fig. 1a). In Fig. 1c, the chemical potential of up-spin $\left(\mu_{\uparrow}\right)$ is lower than that of down-spin $\left(\mu_{\downarrow}\right)$, whereas $\mu_{\uparrow}$ is higher than $\mu_{\downarrow}$ in Fig. 1b. The relative order and the magnitude of the two opposite spin-dependent electrochemical potentials should be self-consistent between the band diagram (energy versus momentum) and their spatial distribution plot (energy versus distance), namely $\mu_{\uparrow}$ should be higher than $\mu_{\downarrow}$ in both Fig. 1b, c. We have drawn the corrected versions of Fig. 1b, c in Fig. 1d (band diagram) and Fig. 1e (spatial variation), respectively, under the same bias condition as Fig. 1b, c. Furthermore, Li et al. ${ }^{1}$ used the absolute values of the electrochemical potentials $\left(\left|\mu_{\uparrow}\right|\right.$ and $\left.\left|\mu_{\downarrow}\right|\right)$ in the band diagram (see Fig. 1b), which is also incorrect since it changes the actual physics depending on the choice of the ground or zero energy level. Generally speaking, which spin state of electrons is more occupied should be governed by the difference $\left(\mu_{\uparrow}-\mu_{\downarrow}\right)$ between $\mu_{\uparrow}$ and $\mu_{\downarrow}$, not by their absolute values. Moreover, unlike what is depicted in Fig. $1 c$, the choice of the reference position for zero potential $(\mu=0)$ is not important for determining which spin state is more occupied (or which spin chemical potential is higher). Since the electrons are injected by the right contact and flow from right to left in Fig. 1c, the chemical potential $\left(\mu_{\mathrm{R}}\right)$ of the right contact is higher than that $\left(\mu_{\mathrm{L}}\right)$ of the left contact (see Fig. 1c). Meanwhile, this also means that in the channel there should be more occupation of the leftgoing electron states (corresponding to up-spin states in a TI

\footnotetext{
${ }^{1}$ Department of Physics and Astronomy, Purdue University, West Lafayette, IN 47907, USA. ${ }^{2}$ Birck Nanotechnology Center, Purdue University, West Lafayette, IN 47907, USA. ${ }^{3}$ Department of Physics and Astronomy, University of Wyoming, Laramie, WY 82071, USA. ${ }^{4}$ School of Electrical and Computer Engineering, Purdue University, West Lafayette, IN 47907, USA. ${ }^{5}$ Center for spintronics, Post-silicon Semiconductor Institute, Korea Institute of Science and Technology (KIST), Seoul 02792, South Korea. ${ }^{6}$ Electrical Engineering and Computer Science, University of California Berkeley, CA 94720, USA. ${ }^{7}$ Department of Physics, The Pennsylvania State University, University Park, Pennsylvania 16802, USA. ${ }^{8}$ Purdue Quantum Science and Engineering Institute, Purdue University, West Lafayette, IN 47907, USA. ${ }^{9}$ WPI-AIMR International Research Center for Materials Sciences, Tohoku University, Sendai 980-8577, Japan. Correspondence and requests for materials should be addressed to J.T. (email: jtian@uwyo.edu)
} 
a

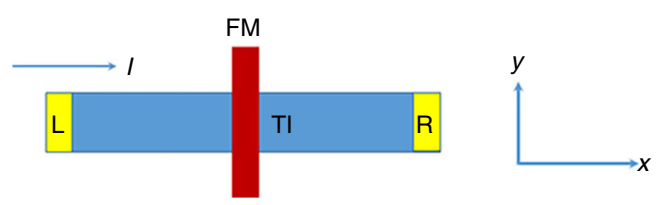

b

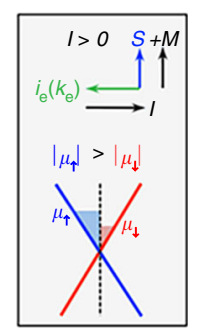

C

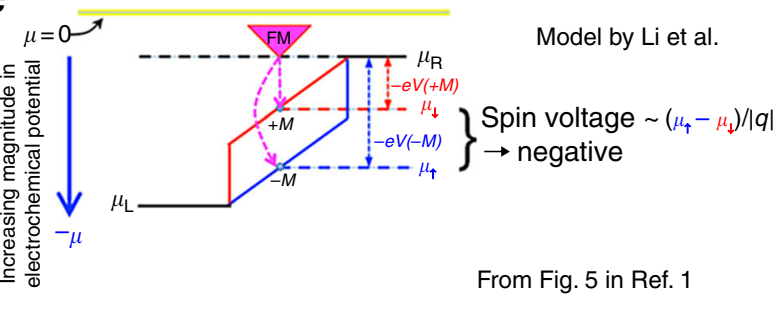

d

e

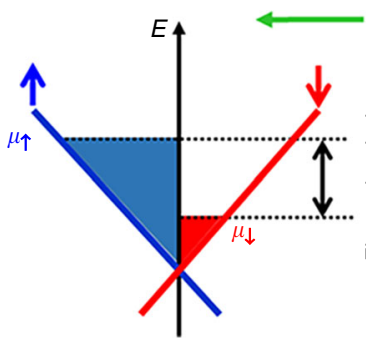

TI surface states band structure

$i_{e}:$ electron flow

Our model

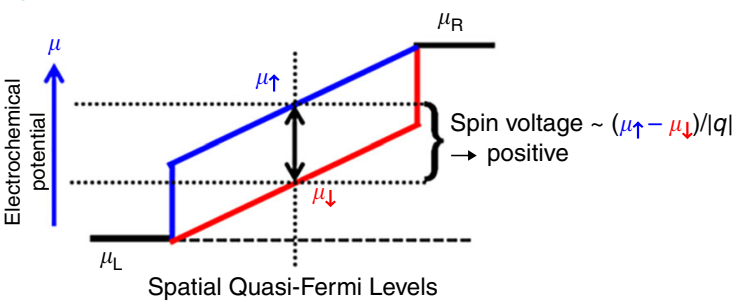

At any point of channel under a charge current flow $\left(i_{\mathrm{e}}\right)$

Fig. 1 Comparison of the models depicting the sign of the spin signals expected from TSS. a The schematic structure of a device with a charge current (I) flowing from left (L) to the right (R); b The band structure diagram of TSS, taken from the left panel shared by Fig. 5b, c in ref. ${ }^{1}$; $\mathbf{c}$ Diagram showing spatially varying spin-dependent electrochemical potential, taken from the top panel of Fig. $5 \mathrm{c}$ in ref. ${ }^{1}$. d, e Our understanding of the spin-dependent electrochemical potentials of TSS in 3D TIs under the same bias current as that shown in $\mathbf{b}, \mathbf{c}$. The chemical potential $\left(\mu_{\uparrow}\right)$ of up spins is higher than that $\left(\mu_{\downarrow}\right)$ of down spins in both $\mathbf{d}$ and $\mathbf{e}$

Table 1 Comparison of the reported results on CISP in 3D Tls measured by spin potentiometry

\begin{tabular}{|c|c|c|c|c|c|}
\hline & Li et al. (refs. ${ }^{1,2}$ ) & Tian et al. (ref. ${ }^{5}$ ) & Dankert et al. (ref. ${ }^{6}$ ) & Lee et al. (ref. ${ }^{7}$ ) & Yang et al. (ref. ${ }^{8}$ ) \\
\hline Charge current direction $\left(I_{c}\right)$ & $+x$ & $+x$ & $+x$ & $+x$ & $+x$ \\
\hline Electron current direction $\left(I_{\mathrm{e}}\right)$ & $-x$ & $-x$ & $-x$ & $-x$ & $-x$ \\
\hline Magnetization direction of FM (M) & $+\mathrm{y}$ & $+y$ & $+\mathrm{y}$ & $+\mathrm{y}$ & $+y$ \\
\hline Sign of spin signal $\left(V_{S}\right)$ & - & + & + & + & + \\
\hline Channel spin polarization direction $\left(\mathrm{s}_{\mathrm{c}}\right)$ & $-y$ & $+y$ & $+\mathrm{y}$ & $+\mathrm{y}$ & $+y$ \\
\hline Spin polarization from TSS (s) & $+y$ & $+y$ & $+y$ & $+\mathrm{y}$ & $+y$ \\
\hline
\end{tabular}

channel with the SML of TSS) than the right-going states (corresponding to the down-spin states), thus $\mu_{\uparrow}$ (equivalently the chemical potential of left-going electrons) should be higher than $\mu_{\downarrow}$ (the chemical potential of right-going electrons), whereas $\mu_{\uparrow}$ is incorrectly drawn to be lower than $\mu_{\downarrow}$ in Fig. 1b. Based on the above arguments, the sign of the spin voltage expected from the corrected potential diagram (seen Fig. 1d, e) in conjunction with their model argument $b$ is opposite to the signal sign on $\mathrm{Bi}_{2} \mathrm{Se}_{3}$ reported by Li et al. Thus, the origin of the CISP observed in their $\mathrm{Bi}_{2} \mathrm{Se}_{3}$ samples is inconsistent with the expected CISP from the TSS.

We further note that $\mathrm{Li}$ et al. ${ }^{1}$ incorrectly stated that another experiment on $(\mathrm{Bi}, \mathrm{Sb})_{2} \mathrm{Te}_{3}$ by Lee et al. ${ }^{7}$ (which is ref. 29 in ref. ${ }^{1}$ ) gave a consistent sign as theirs, whereas it is opposite. We point out that the sign reported by Lee et al. ${ }^{7}$ is consistent with several other reports (see, refs. ${ }^{5,6,8}$ ) while opposite to Li et al. ${ }^{1,2}$, as summarized in Table 1.

Li et al. ${ }^{1}$ also attempted to infer the origin of CISP in their $\mathrm{Bi}_{2} \mathrm{Se}_{3}$ by making a comparison with SOC semiconductor InAs, where a Rashba-type 2DEG normally exists on the surface. We caution that such a comparison may not be sufficient to draw a conclusion on the origin of CISP. For example, it is known that, depending on the direction of the effective electric field (potential gradient) perpendicular to the $2 \mathrm{DEG}^{9-11,12}$, the spin helicity of the outer Rashba band (which dominates the signals measured in transport) can be either opposite to or the same as that of TSS. Without a careful consideration of various parameters (e.g., an independent determination of the spin texture, for example by ARPES, and consideration of capping surfaces, interfaces, etc.) of their samples, spin potentiometric measurements in their InAs sample cannot provide an unambiguous "calibration" to determine the direction of the CISP measured by spin potentiometry in their $\mathrm{Bi}_{2} \mathrm{Se}_{3}$.

Further complications can arise from the fact that in addition to the nontrivial spin-momentum-locked $\mathrm{TSS}, \mathrm{Bi}_{2} \mathrm{Se}_{3}$ often contains multiple bands and conducting channels with spin-orbit coupling that can affect CISP. For example, the trivial surface 2DEG derived from bulk states and often observed by $\mathrm{ARPES}^{15}$ in 
$\mathrm{Bi}_{2} \mathrm{Se}_{3}$ possesses strong Rashba-type spin-orbit coupling and typically has two fermi surfaces with opposite spin helicities ${ }^{12}$. We note that the model (Fig. 1d, e) we developed is only for TSS in the ideal case of bulk-insulating TI materials where the Fermi level is inside the bulk band gap. If the TI samples have metallic bulk with their Fermi levels located in the conduction band where the multiple bands coexist, our model may not be sufficient to determine the sources of the measured CISP.

In conclusion, the model used by $\mathrm{Li}$ et al. ${ }^{1}$ is erroneous and inconsistent with their TSS band diagram, and also inconsistent with the expected CISP due to TSS. The sign of the CISP signal experimentally observed in their $\mathrm{Bi}_{2} \mathrm{Se}_{3}$ is opposite to that predicted by a corrected model based on TSS and that observed in other experiments on various TI materials ${ }^{5-8}$. Owing to the existence of multiple bands in the bulk-metallic TI samples, the source of the measured CISP can be complicated, possibly involving competition between different bands (e.g., TSS and Rashba bands). Identifying the origin of CISP in bulk-metallic TI samples such as the $\mathrm{Bi}_{2} \mathrm{Se}_{3}$ used by $\mathrm{Li}$ et al. ${ }^{1}$ may require analysis beyond the simple model (Fig. 1c, d) we developed.

\section{Data availability}

All relevant data are available from the authors upon request.

Received: 23 April 2018 Accepted: 28 February 2019

Published online: 01 April 2019

\section{References}

1. Li, C. H., van 't Erve, O. M. J., Rajput, S., Li, L. \& Jonker, B. T. Direct comparison of current-induced spin polarization in topological insulator $\mathrm{Bi}_{2} \mathrm{Se}_{3}$ and InAs Rashba states. Nat. Commun. 7, 13518 (2016).

2. $\mathrm{Li}, \mathrm{C}$. H. et al. Electrical detection of charge-current-induced spin polarization due to spin-momentum locking in $\mathrm{Bi}_{2} \mathrm{Se}_{3}$. Nat. Nanotechnol. 9, 218-224 (2014).

3. Tang, J. et al. Electrical detection of spin-polarized surface states conduction in $\left(\mathrm{Bi}_{0.53} \mathrm{Sb}_{0.47}\right)_{2} \mathrm{Te}_{3}$ topological insulator. Nano. Lett. 14, 5423-5429 (2014).

4. Ando, Y. et al. Electrical detection of the spin polarization due to charge flow in the surface state of the topological insulator $\mathrm{Bi}_{1.5} \mathrm{Sb}_{0.5} \mathrm{Te}_{1.7} \mathrm{Se}_{1.3}$. Nano. Lett. 14, 6226-6230 (2014).

5. Tian, J. et al. Electrical injection and detection of spin-polarized currents in topological insulator $\mathrm{Bi}_{2} \mathrm{Te}_{2}$ Se. Sci. Rep. 5, 14293 (2015).

6. Dankert, A., Geurs, J., Kamalakar, M. V. \& Dash, S. P. Room temperature electrical detection of spin polarized currents in topological insulators. Nano. Lett. 15, 7976 (2015).

7. Lee, J. S., Richardella, A., Hickey, D. R., Mkhoyan, K. A. \& Samarth, N. Mapping the chemical potential dependence of current-induced spin polarization in a topological insulator. Phys. Rev. B 92, 155312 (2015).

8. Yang, F. et al. Switching of charge-current-induced spin polarization in the topological insulator BiSbTeSe 2 . Phys. Rev. B 94, 075304 (2016).
9. Hammar, P. R. \& Johnson, M. Potentiometric measurements of the spinsplit subbands in a two-dimensional electron gas. Phys. Rev. B. 61, 7207 (2000).

10. Johnson, M. Spin injection and detection in a ferromagnetic metal/2DEG structure. Phys. E 10, 472-477 (2001).

11. Park, Y. H. et al. Observation of spin-orbit interaction parameter over a wide temperature range using potentiometric measurement. IEEE Trans. Magn. 46, 1562 (2010).

12. Park, Y. H. et al. Observation of gate-controlled spin-orbit interaction using a ferromagnetic detector. J. Appl. Phys. 111, 07C317 (2012).

13. Hong, S., Diep, V., Datta, S. \& Chen, Y. P. Modeling potentiometric measurements in topological insulators including parallel channels. Phys. Rev. $B$ 86, 085131 (2012).

14. Sayed, S., Hong, S. \& Datta, S. Multi-terminal spin valve on channels with spin-momentum locking. Sci. Rep. 6, 35658 (2016).

15. Bahramy, M. S. et al. Emergent quantum confinement at topological insulator surfaces. Nat. Commun. 3, 1159 (2012).

\section{Acknowledgements}

This work was support by the DARPA (Defense Advanced Research Projects Agency) MESO (Mesodynamic Architectures) program (grant N66001-11-1-4107) and NSF (National Science Foundation, grant 1641101). S.H. acknowledges the KIST Institutional Program.

\section{Author contributions}

Y.P.C., S.D. and N.S. conceived the idea. J.T., S.H., S.S. and J.S.L. carried out the analyses, J.T., S.H., S.S. and Y.P.C. wrote the paper with feedback from all co-authors.

\section{Additional information}

Competing interests: The authors declare no competing interests.

Reprints and permission information is available online at http://npg.nature.com/ reprintsandpermissions/

Journal peer review information: Nature Communications thanks the anonymous reviewer(s) for their contribution to the peer review of this work.

Publisher's note: Springer Nature remains neutral with regard to jurisdictional claims in published maps and institutional affiliations.

\begin{abstract}
cc) (1) Open Access This article is licensed under a Creative Commons Attribution 4.0 International License, which permits use, sharing, adaptation, distribution and reproduction in any medium or format, as long as you give appropriate credit to the original author(s) and the source, provide a link to the Creative Commons license, and indicate if changes were made. The images or other third party material in this article are included in the article's Creative Commons license, unless indicated otherwise in a credit line to the material. If material is not included in the article's Creative Commons license and your intended use is not permitted by statutory regulation or exceeds the permitted use, you will need to obtain permission directly from the copyright holder. To view a copy of this license, visit http://creativecommons.org/ licenses/by/4.0/.
\end{abstract}

(C) The Author(s) 2019 